\title{
Transferring a poor quality embryo with a good quality embryo benefits poor prognosis patients: a retrospective study using propensity score matching
}

\author{
wenjie wang ${ }^{1}$, Jiali $\mathrm{Cai}^{1}$, Lanlan $\mathrm{Liu}^{1}$, Yingpei $\mathrm{Xu}^{1}$, Zhenfang $\mathrm{Liu}^{1}$, Jinghua Chen ${ }^{1}$, \\ Xiaoming Jiang ${ }^{1}$, Xiaohua Sun $^{1}$, and Jianzhi Ren ${ }^{1}$ \\ ${ }^{1}$ Xiamen University Affiliated Chenggong Hospital
}

July 9, 2020

\begin{abstract}
Objective: To evaluate the effect of transferring a poor quality embryo (PQE) with a good quality embryo (GQE) on the outcomes in poor prognosis patients. Design: Retrospective cohort study. Setting: University-affiliated hospital. Population: A total of 5,037 FBT cycles between January 2012 and May 2019. Methods: Single FBT with GQE were defined as group G and double FBT with GQE plus PQE were defined as group GP. Propensity score matching was applied to control for potential confounders. Multivariate generalized estimating equations (GEE) models were used to identify the association between the effect of an additional PQE and outcomes. Main Outcome Measures: Live births and multiple pregnancies. Results: Group GP resulted in a significantly higher live birth rate (LBR) than group $\mathrm{G}$ in women aged 35 and over and in women who received over 3 cycles of embryo transfer (ET) (48.1\% vs $27.2 \%$, OR:2.56, 95\% CI: $1.3-5.03$ and $46.6 \%$ vs $35.4 \%$, OR:1.6, 95\% CI: $1.09-2.35$ ), while LBR were statistically similar between two groups in women under 35 and in women who received less than 3 cycles of ET (48.7\% vs $43.9 \%$, OR:1.22, 95\% CI: $0.93-1.59$ and $48.3 \%$ vs $41.4 \%$, OR:1.33, 95\% CI: 0.96-1.85). Notably, group GP were found a consistent significantly higher multiple pregnancy rate than group G. Conclusions: The transfer of an additional PQE with a GQE increases live births in poor prognosis patients. Funding:None. Keywords: poor quality embryo, good quality embryo, LBR, poor prognosis patients
\end{abstract}

\section{Introduction}

The primary goal of assisted reproduction technology (ART) is the birth of one single healthy child at a time, while multiple pregnancies were considered as the most serious adverse outcome related to ART, associated with increased risks of maternal and fetal morbidity ${ }^{1}$. Risks associated with multiple pregnancies include higher rates of cerebral palsy, preterm delivery, and low birth weight, perinatal death compared with singleton pregnancies ${ }^{2}$. The most effective way to minimize the incidence of multiple pregnancies is single embryo transfer (SET).

The developments of extended embryo culture and the introduction of vitrification have allowed the widespread application of SET at the blastocyst stage and surplus blastocysts to be vitrified for subsequent frozen-thawed blastocyst transfer (FBT) ${ }^{3,4}$. While single blastocyst transfer (SBT) is commonly adopted in fresh cycles, double blastocyst transfer (DBT) remains preferable in frozen-thawed blastocyst transfer (FBT) cycles due to the concerns of reduction of live birth rate (LBR) ${ }^{5}$. Previous studies reported conflicting results when comparing clinical pregnancy rate (PR) and LBR of SBT with DBT in frozenthawed cycles. Some investigators demonstrated no significant differences in PR and LBR between SBT and $\mathrm{DBT}^{6,7}$, while some studies indicated SBT met a lower PR and a lower LBR compared to DBT ${ }^{5,8}$. Nevertheless, all of these studies supported that SBT significantly decreased the multiple pregnancy rate (MPR). 
On the other hand, embryo quality has been reported as a major predictor of IVF success rates ${ }^{9}$. It is well established that poor quality embryo (PQE) has a lower implantation potential than good quality embryo (GQE). However, we may confront a dilemma in clinical practice, whether it would be worthwhile to transfer an additional PQE with a GQE when the decision concerns embryo quality and number of embryos. In particular, patients in certain situations (advanced age, failed repeatedly) commonly resulted in only PQEs with one or no GQE. Moreover, these patients with poor prognosis were associated with limited chance of conception and increased risks of obstetrical and neonatal complications ${ }^{10,11}$.

Therefore, the objective of this study was to evaluate the effect of DBT with one GQE plus one PQE on outcomes in women undergoing FBT stratified by age and cycles of ET. This information would be beneficial for clinicians making transfer policy for patients with different prognosis. It will also promote the reduction of overall MPR without impairment of LPR.

\section{Materials and Methods}

\section{Study design and patients}

A retrospective study was performed at Xiamen University Affiliated Chenggong Hospital. All the frozenthawed blastocyst transfer (FBT) cycles in the period between January 2012 and May 2019 were included. Exclusion criteria were: (a) blastulation on day 7 (b) Blastocysts derived from vitrified oocytes or vitrified cleavages. Cycles with missing data and women lost to follow-up were secondarily excluded. Patients undergoing single FBT with one GQE were defined as group G and double FBT with one GQE plus one PQE were defined as group GP. Institutional Review Board approval was obtained from the Ethics Committee of the Medical College of Xiamen University.

\section{Treatment protocol and embryo quality assessment}

In stimulation cycles, all patients were treated with agonist or antagonist protocol with the use of FSH or hMG as previously described ${ }^{12}$. The initial and ongoing dosage was adjusted according to the patient's age, antral follicle count (AFC), BMI, and ovarian response. Ovarian response was monitored by means of transvaginal ultrasound measurements of follicular growth and serum E2 level every 1-3 days. When at least one follicle reached a mean diameter of $18 \mathrm{~mm}$ as determined by ultrasound, hCG was administrated. Oocyte retrieval was scheduled for 34-36 hours after hCG injection and was carried out under transvaginal ultrasound guidance.

Conventional IVF or ICSI was carried out depending on semen parameters and previous fertilization histories. In IVF cycles, cumulus-oocyte complexes were inseminated with approximate $1.5-3 \mathrm{X} 10^{5}$ progressively motile spermatozoa in fertilization culture medium (K-SIFM, Cook) for $4 \mathrm{~h}$. Oocytes for ICSI were denuded $2 \mathrm{~h}$ after ovum pickup, and sperm microinjection was performed $4 \mathrm{~h}$ after retrieval. Fertilization was checked about $17 \mathrm{~h}$ post insemination/injection and was determined by the presence of two pronuclei $(2 \mathrm{PN})$.

All embryos were cultured under mineral oil in traditional incubators (C200, Labotect) at 37, 6\% CO2, $5 \%$ O2. Cook IVF media (Cook Medical) was used for cleavage-stage embryos (K-SICM) and blastocysts (K-SIBM) culture in the form of microdroplet of $20 \mathrm{ul}$. On day 3, evaluation of embryo quality included the number of blastomere, the degrees of fragmentation and the uniformity of blastomeres. Cleavages were determined for fresh embryo transfer or blastocyst culture, and then were placed in blastocyst culture medium(K-SIBM). The quality of blastocysts were evaluated on Day 5 or Day 6 based on the Gardner and Schoolcraft grading system, and the score was dependent on blastocyst expansion, inner cell mass (ICM) development and trophectoderm (TE) appearance ${ }^{13}$. Good quality embryos were blastocysts graded as AA, $\mathrm{AB}, \mathrm{BA}$ and $\mathrm{BB}$ with expansion grade [?]3, while poor quality embryos were those defined as $\mathrm{AC}, \mathrm{CA}, \mathrm{BC}$, $\mathrm{CB}$ and $\mathrm{CC}$ with expansion grade [?]3. In addition, Top quality embryo (TQE)were blastocysts graded as $\mathrm{AA}, \mathrm{AB}$ and $\mathrm{BA}$ with expansion grade [?]4. Blastocysts were determined to be transferred on day 5 or vitrified for subsequent transfer. Blastocysts with poor morphological score ([?]4CC) or low expansion grade (grades 1-2) were not considered for vitrification or transfer.

\section{Vitrification and thawing}


For vitrification, the Cryotop method was carried out as described by Kuwayama ${ }^{14}$. Briefly, blastocysts were equilibrated for 3-5 min in equilibration solution (ES:7.5\% dimethyl sulfoxide and 7.5\% ethylene glycol), and were then placed into in vitrification solution (VS:15\% dimethyl sulfoxide, $15 \%$ ethylene glycol, $10 \mathrm{mg} / \mathrm{mL}$ Ficoll-70, and 0.6 M sucrose). After 30-40 s in VS, embryos were transferred on the Cryotop strip and plunged into liquid nitrogen immediately. For thawing, blastocysts were directly immersed into thawing solution (TS) containing $1 \mathrm{M}$ sucrose at 37 for $1 \mathrm{~min}$, then was sequentially incubated in each of the following solutions for 3 minutes: $0.5 \mathrm{M}$ sucrose, $0.25 \mathrm{M}$ sucrose and sucrose-free TS. Then the embryos were placed in blastocyst culture medium (K-SIBM, Cook) and cultured in an incubator at 37 with $6 \%$ CO2 until transfer. Survival of thawed embryos were assessed under an inverted microscope depending on whether blastocysts showed a severely damaged cellular content or not.

\section{Endometrial preparation and embryo transfer}

Three main types of endometrial preparation protocols were applied: the natural cycle (NC), hormone replacement treatment (HRT) cycle with or without GnRH downregulation. In NC cycles, growth of follicles were monitored under

transvaginal ultrasonography from cycle day 9 to 11 . LH and estradiol were measured every 3 days after the diameter of leading follicle [?] $1.4 \mathrm{~mm}$. Intramuscular progesterone injections (40 mg/day) were started at the day of ovulation and FBT was scheduled on the 5th day after ovulation. HRT was performed with 6-8 $\mathrm{mg}$ oral estradiol valerate daily from cycle day 1 to day 14. Progesterone injection (60-100 mg) was administrated as soon as the endometrial thickness reached 7-8 $\mathrm{mm}$ and then FBT was scheduled after 5 days of progesterone therapy. As for HRT with GnRH- downregulation cycles, GnRH agonist was initiated on day 1 of the menstrual cycle. And on day 1 of subsequent menstruation, estrogen stimulation was started as HRT cycles without GnRH agonist. Embryo transfer was performed with a Guardia Access Embryo Transfer catheter (K-JETS-7019-SIVF, Cook, IN, USA) under transabdominal ultrasound guidance. Luteal support continued until 10 weeks of pregnancy.

\section{Statistical analysis}

The primary outcome was live birth rate (LBR). Secondary outcomes of the study included clinical pregnancy rate(PR), miscarriage rate (MR) and multiple pregnancy rate (MPR).

The baseline characteristics were compared between the two groups. The normality of continuous variables was examined by normality plots and Shapiro-Wilk test. Since none of the Continuous variables studied demonstrated normal distribution by both tests, they are presented as medians (first quartile, third quartile), while categorical variables are presented as n (\%). Continuous variables were analyzed by Mann-Whitney U test, and categorical variables were analyzed using Chi-square test or Fisher's exact test. All values were two tailed and $\mathrm{P}<0.05$ was considered to be significant. All analysises were performed by using SPSS (version $22, \mathrm{IBM})$.

As two groups were not randomly assigned in clinical practice, potential confounders and selection biases were accounted for by propensity score matching ${ }^{15}$. Propensity scores were calculated using logistic regression based on potential variables related to the outcome ${ }^{16}$. The variables included maternal age, paternal age, maternal BMI, parity, gravidity, duration of infertility, cause of infertility, baseline FSH, antral follicle count (AFC), ovarian stimulation protocol, insemination methods, endometrial preparation protocol, endometrial thickness, number of blastocyst vitrified, cycles of ET, day of blastocyst transferred and the proportion of using top quality blastocysts. A one-to-one nearest neighbor matching method without replacement was performed to match data between group G and group GP with a caliper width equal to $0.03{ }^{17}$. In order to investigate the effect of group GP in women aged 35 and over and in women received at least 3 cycles of ET, two groups were stratified by age and ET order, and PS matching of each subgroup was performed separately. PS matching was performed by using MatchIt package in R software.

A generalized estimating equations (GEE) model was conducted to evaluate the association between the effect of an additional PQE and outcomes due to including patients contributing multiple cycles ${ }^{8}$. To 
further verify the results, multivariate GEE models were performed using pre-matching data to adjust for aforementioned confounders.

\section{Results}

A total of 5,037 women were included in this study. Group G consisted of 4,484 patients and Group GP included 553 patients. After propensity score matching, 520 patients in group $\mathrm{G}$ were matched by their counterparts in group GP.

Patients' overall demographics and baseline IVF characteristics were presented in table 1 (left panel). Significant differences were observed in terms of paternal age, AFC, duration of infertility, No. of blastocysts vitrified, ovarian stimulation protocol, endometrial preparation protocol, cycles of ET, day of blastocyst transferred and the proportion of using top quality blastocysts between two groups $(\mathrm{P}<0.05)$. Comparison after PS matching was also listed in table 1 (right panel), all the baseline characteristics became very comparable between two groups $(\mathrm{P}>0.05)$. The distributions of the standard differences before and after PS matching were plotted (Figure S1). Standard difference $<0.1$ was used as the threshold to indicate a negligible difference in the prevalence of a covariate between exposure groups.

Table 2 illustrates the outcomes of both groups before and after PS matching. Group GP achieved significantly higher PR (57.3\%vs47.3\%, OR:1.51, 95\% CI: $1.18-1.93)$, LBR (47.9\% vs $41 \%$, OR:1.33, $95 \%$ CI: 1.04-1.7) and MPR (30.5\%vs2.4\%, OR: 17.49, 95\% CI: 7.49-40.81) than group G after PS matching. MR for group GP were similar to group G (15.4\% vs $13.4 \%$, OR: $1.18,95 \%$ CI: $0.73-1.9)$.

The outcomes of both groups stratified by age using a cutoff of 35 years old were displayed in table 3 . After matching, in women less than 35 years of age, PR (58\% vs $50.1 \%$, OR:1.38, 95\% CI: $1.05-1.82)$ and MPR (31.7\% vs 1.9\%, OR:23.81, 95\% CI: 8.54-66.43) were significantly higher in group GP than in group G. However, there were no significant differences in MR and LBR between two groups (31.6\% vs $27.4 \%$ and $48.7 \%$ vs $43.9 \%$, respectively). Interestingly, as for women 35 years of age and over, not only PR and MPR, but also LBR (48.1\%vs27.2\%, OR:2.56, 95\% CI: 1.3-5.03) were found significantly higher in group GP than in group G. Adjusted OR for multiple pregnancy in women aged 35 and over before PS matching was not given, because multivariate GEE model was not available when the incidence of multiple pregnancy is low in relation to 21 variables used in the adjustment model.

Comparisons of two groups stratified by cycles of ET were listed in table 4. For patients who received ET less than 3 cycles, group GP had no differences in PR, MR and LBR compared to group G. However, both PR ( $56.5 \%$ vs $42.2 \%$, OR:1.79, 95\% CI: $1.22-2.61)$ and LBR (46.6\% vs $35.4 \%$, OR:1.6, 95\% CI: $1.09-2.35)$ were observed statistically higher in group GP when compared to group G in patients undergoing at least 3 times embryo transfer. MPR were consistently significantly higher in group GP than in group G $(31.7 \%$ vs $5.6 \%$, OR:7.97, 95\% CI: 3.6-17.63) regardless of ET cycles.

\section{Discussion}

This study indicated that double FBT with one GQE plus one PQE increased LBRs mainly in patients aged 35 and over and in patients received at least 3 cycles of ET when compared with single FBT with only one GQE. Notably, MPR was significantly higher in group GP than in group G regardless of age or prior ET cycles. MR remained comparable no matter two groups were stratified by age or cycles of ET or not.

Several studies have evaluated the impact of double blastocyst transfer (DBT) on clinical outcomes compared with single blastocyst transfer (SBT). Dobson and colleagues demonstrated DBT with one PQE plus one GQE does not increase LBR but increases MPR when compared with SBT with a TQE during fresh or frozen blastocyst transfer ${ }^{18}$. However, the only confounder they have adjusted for OR was age. There were likely some other factors which would confound for the results as other studies have reported ${ }^{19,20}$. Our study also resulted in no difference in LBR (OR:0.93, 95\%CI:0.77-1.11) between two groups when only adjusted for age before PS matching. Wintner and colleagues also concluded no statistically significant difference in LBR between these two groups ${ }^{21}$. However, their study was performed in fresh cycles with ET at cleavage stage or blastocyst stage, which might differ from FBT ${ }^{22}$. On the other hand, El-Danasouri and colleagues found 
that transferring an impaired quality embryo along with a good quality embryo significantly lowered both the pregnancy rate and implantation rate, than transferring the good quality embryo alone ${ }^{23}$. However, their study did not demonstrate a statistically significant difference. In contrast to their reports, some investigators demonstrated that transferring two blastocysts in FBT cycles lead to a higher PR, a higher LBR and no doubt a higher MPR ${ }^{5,8}$, which is consistent to our results. In spite of different results in LBR, aforementioned studies concluded consistently that double FBT achieved a significantly higher MPR than single FBT.

Two prior studies have compared the outcomes of DBT with SBT in advanced maternal age. One study found that DBT resulted in a higher live birth than SBT for women aged 35 years and over undergoing vitrified-warmed cycles ${ }^{6}$. Another study indicated eSBT is associated with similar LBRs compared to the entire DBT cohort, but the subgroup of women who had elective DBT achieved a higher LBR and a higher MP in advanced maternal age ${ }^{24}$. In accordance to their results, our study also observed DBT with one GQE plus one PQE achieved a higher LBR than SBT with only one GQE in women aged 35 and over but not in women under 35 years of age. Our data showed that DBT maintained a similar PR, MPR, MR and LBR between the subgroups stratified by 35 years of age, whereas SBT resulted in a significantly lower LBR due to a lower trend in PR and a statistically higher miscarriage rate. The miscarriage rate significantly increased may possibly because the prevalence of aneuploidy rose along with maternal age ${ }^{25}$. Poor quality blastocysts owing to age-related decline in oocyte quality were assessed $25.5 \%$ euploid compared with $56 \%$ of the top-quality blastocysts ${ }^{26}$. Despite higher incidence of aneuploidy and a lower implantation than good quality blastocyst, poor quality blastocysts still contributed an overall increase of live births ${ }^{27}$.

Few studies have reported the comparison of double FBT versus single FBT in women failed repeatedly. A study performed by McLernon and his colleagues to develop the prediction model to estimate the chances of a live birth implied the association between number of complete cycles with live birth ${ }^{28}$. Although it didn't meet the definition of recurrent implantation failure, patients underwent at least 3 cycles of embryo transfer implied difficulties of conception with various reasons ${ }^{10}$. Our study found that DBT of PQE with GQE achieved a significantly higher LBR in women received over 3 cycles of ET due to a higher PR and a similar MR compared with SBT. The possible explanation of a higher PR might be that the promotion of interaction between embryo and endometrium by an additional embryo led to a more favorable endometrial receptivity ${ }^{29}$. Anyway, it indicated that poor quality blastocyst had implantation potential to provide additional increase of LBR and should be vitrified for future use in patients with poor prognosis.

A main strength of our study is that it included the largest number of patients on this topic to-date. Second, PS matching was conducted to control for potential confounders which might have effects on the outcomes. It has been proofed that PS matching provides an approach to mimic random assignment as RCT and is superior to conventional regression-based methods in a real world observational study ${ }^{15}$. Third, the comparisons were not only performed in overall groups, but were also explored in advanced maternal age and in women received at least 3 cycles of ET.

Our study was limited by its retrospectively observational design, and patients' information were previously recorded by hospital with some missing data. Though PS matching was performed to evaluate the effects of DBT with mixed quality embryo independently from other confounders, the sample decreased after PS and the loss of unmatched cases might have unforeseen effects. Accordingly, results by multivariable GEE models to adjust potential confounders before PS matching was presented relatively.

\section{Conclusion}

Based on these results, we support SBT with one GQE in patients with good prognosis due to non-difference in LBR but a significantly lower MPR. The transfer of an additional PQE with a GQE increases live births in poor prognosis patients. However, it should still be cautious since they are associated with higher risks of multiple pregnancies.

\section{Declarations}




\section{Authors' roles}

WW contributed to statistical analysis and interpretation of data and drafting of the manuscript. JC and LL conceived and designed this study, performed statistical analysis and participated in the discussion. YX, ZL and JC were involved in acquisition, analysis and interpretation of data. XJ, XS and RJ participated in the discussion and critically revised the manuscript. All authors have read and approved the final version of the manuscript.

\section{Acknowledgements}

The authors would like to thank to Xinli Wang for support on data processing.

\section{Funding}

None

\section{Conflict of interest}

None declared.

\section{Ethics approval}

Institutional Review Board approval was obtained from the Ethics Committee of the Medical College of Xiamen University on 10 December 2018 (2019-010).

\section{References}

1. Sullivan EA, Wang YA, Hayward I, Chambers GM, Illingworth P, McBain J, et al. Single embryo transfer reduces the risk of perinatal mortality, a population study. Human reproduction (Oxford, England). 2012 Dec;27(12):3609-15.

2. McLernon DJ, Harrild K, Bergh C, Davies MJ, de Neubourg D, Dumoulin JC, et al. Clinical effectiveness of elective single versus double embryo transfer: meta-analysis of individual patient data from randomised trials. BMJ (Clinical research ed). 2010 Dec 21;341:c6945.

3. Rienzi L, Gracia C, Maggiulli R, LaBarbera AR, Kaser DJ, Ubaldi FM, et al. Oocyte, embryo and blastocyst cryopreservation in ART: systematic review and meta-analysis comparing slow-freezing versus vitrification to produce evidence for the development of global guidance. Human reproduction update. 2017 Mar 1;23(2):139-55.

4. Wirleitner B, Schuff M, Stecher A, Murtinger M, Vanderzwalmen P. Pregnancy and birth outcomes following fresh or vitrified embryo transfer according to blastocyst morphology and expansion stage, and culturing strategy for delayed development. Human reproduction (Oxford, England). 2016 Aug;31(8):168595.

5. Berin I, McLellan ST, Macklin EA, Toth TL, Wright DL. Frozen-thawed embryo transfer cycles: clinical outcomes of single and double blastocyst transfers. Journal of assisted reproduction and genetics. 2011 Jul;28(7):575-81.

6. Eum JH, Park JK, Kim SY, Paek SK, Seok HH, Chang EM, et al. Clinical outcomes of single versus double blastocyst transfer in fresh and vitrified-warmed cycles. Clinical and experimental reproductive medicine. 2016 Sep;43(3):164-8.

7. Racca A, Drakopoulos P, Van Landuyt L, Willem C, Santos-Ribeiro S, Tournaye H, et al. Single and double embryo transfer provide similar live birth rates in frozen cycles. Gynecological endocrinology : the official journal of the International Society of Gynecological Endocrinology. 2020 Mar 3:1-5.

8. Devine K, Connell MT, Richter KS, Ramirez CI, Levens ED, DeCherney AH, et al. Single vitrified blastocyst transfer maximizes liveborn children per embryo while minimizing preterm birth. Fertil Steril. 2015 Jun;103(6):1454-60.e1. 
9. Zhang J, Huang J, Liu H, Wang B, Yang X, Shen X, et al. The impact of embryo quality on singleton birthweight in vitrified-thawed single blastocyst transfer cycles. Human reproduction (Oxford, England). 2020 Feb 29;35(2):308-16.

10. Bashiri A, Halper KI, Orvieto R. Recurrent Implantation Failure-update overview on etiology, diagnosis, treatment and future directions. Reproductive biology and endocrinology : RB\&E. 2018 Dec 5;16(1):121.

11. Prapas N, Kalogiannidis I, Prapas I, Xiromeritis P, Karagiannidis A, Makedos G. Twin gestation in older women: antepartum, intrapartum complications, and perinatal outcomes. Archives of gynecology and obstetrics. 2006 Feb;273(5):293-7.

12. Cai J, Liu L, Zhang J, Qiu H, Jiang X, Li P, et al. Low body mass index compromises live birth rate in fresh transfer in vitro fertilization cycles: a retrospective study in a Chinese population. Fertil Steril. 2017 Feb;107(2):422-9.e2.

13. Gardner DK, Schoolcraft WB. Culture and transfer of human blastocysts. Current opinion in obstetrics \& gynecology. 1999 Jun;11(3):307-11.

14. Kuwayama M. Highly efficient vitrification for cryopreservation of human oocytes and embryos: the Cryotop method. Theriogenology. 2007 Jan 1;67(1):73-80.

15. Agoritsas T, Merglen A, Shah ND, O'Donnell M, Guyatt GH. Adjusted Analyses in Studies Addressing Therapy and Harm: Users' Guides to the Medical Literature. Jama. 2017 Feb 21;317(7):748-59.

16. Austin PC. An Introduction to Propensity Score Methods for Reducing the Effects of Confounding in Observational Studies. Multivariate behavioral research. 2011a May;46(3):399-424.

17. Austin PC. Optimal caliper widths for propensity-score matching when estimating differences in means and differences in proportions in observational studies. Pharmaceutical statistics. 2011b Mar-Apr;10(2):15061.

18. Dobson SJA, Lao MT, Michael E, Varghese AC, Jayaprakasan K. Effect of transfer of a poor quality embryo along with a top quality embryo on the outcome during fresh and frozen in vitro fertilization cycles. Fertil Steril. 2018 Sep;110(4):655-60.

19. Veleva Z, Orava M, Nuojua-Huttunen S, Tapanainen JS, Martikainen H. Factors affecting the outcome of frozen-thawed embryo transfer. Human reproduction (Oxford, England). 2013 Sep;28(9):2425-31.

20. Ahlström A, Westin C, Wikland M, Hardarson T. Prediction of live birth in frozen-thawed single blastocyst transfer cycles by pre-freeze and post-thaw morphology. Human reproduction (Oxford, England). 2013 May;28(5):1199-209.

21. Wintner EM, Hershko-Klement A, Tzadikevitch K, Ghetler Y, Gonen O, Wintner O, et al. Does the transfer of a poor quality embryo together with a good quality embryo affect the In Vitro Fertilization (IVF) outcome? Journal of ovarian research. 2017 Jan 13;10(1):2.

22. Wei D, Liu JY, Sun Y, Shi Y, Zhang B, Liu JQ, et al. Frozen versus fresh single blastocyst transfer in ovulatory women: a multicentre, randomised controlled trial. Lancet (London, England). 2019 Mar 30;393(10178):1310-8.

23. El-Danasouri I, Sterzik K, Rinaldi L, Pacchiarotti A, DeSanto M, Selman H. Effect of transferring a morphologically impaired embryo with a good quality embryo on the pregnancy and implantation rates. European review for medical and pharmacological sciences. 2016;20(3):394-8.

24. Tannus S, Son WY, Dahan MH. Elective single blastocyst transfer in advanced maternal age. Journal of assisted reproduction and genetics. 2017 Jun;34(6):741-8.

25. Franasiak JM, Forman EJ, Hong KH, Werner MD, Upham KM, Treff NR, et al. The nature of aneuploidy with increasing age of the female partner: a review of 15,169 consecutive trophectoderm biopsies evaluated 
with comprehensive chromosomal screening. Fertil Steril. 2014 Mar;101(3):656-63.e1.

26. Capalbo A, Rienzi L, Cimadomo D, Maggiulli R, Elliott T, Wright G, et al. Correlation between standard blastocyst morphology, euploidy and implantation: an observational study in two centers involving 956 screened blastocysts. Human reproduction (Oxford, England). 2014 Jun;29(6):1173-81.

27. Cimadomo D, Soscia D, Vaiarelli A, Maggiulli R, Capalbo A, Ubaldi FM, et al. Looking past the appearance: a comprehensive description of the clinical contribution of poor-quality blastocysts to increase live birth rates during cycles with aneuploidy testing. Human reproduction (Oxford, England). 2019 Jul 8;34(7):1206-14.

28. McLernon DJ, Steyerberg EW, Te Velde ER, Lee AJ, Bhattacharya S. Predicting the chances of a live birth after one or more complete cycles of in vitro fertilisation: population based study of linked cycle data from 113873 women. BMJ (Clinical research ed). 2016 Nov 16;355:i5735.

29. Kurian NK, Modi D. Extracellular vesicle mediated embryo-endometrial cross talk during implantation and in pregnancy. Journal of assisted reproduction and genetics. 2019 Feb;36(2):189-98.

\section{Hosted file}

table.docx available at https://authorea.com/users/341313/articles/468254-transferringa-poor-quality-embryo-with-a-good-quality-embryo-benefits-poor-prognosis-patients-aretrospective-study-using-propensity-score-matching 